\title{
FINITE ANTI-PLANE SHEAR OF A SEMI-INFINITE STRIP SUBJECT TO A SELF-EQUILIBRATED END TRACTION*
}

\author{
BY \\ C. O. HORGAN AND R. ABEYARATNE \\ Michigan State University
}

1. Introduction. In a recent paper [1], a version of Saint-Venant's principle was formulated and established for finite elastostatics. We refer to [1] and the references cited therein for a discussion of the issues underlying Saint-Venant's principle both for linear and nonlinear elasticity. (A review of recent work on principles of Saint-Venant type is given in [2]. For a discussion of earlier results in the linear theory of elasticity, see [3].) Some important new features which arise in the latter context are: (i) it is no longer sufficient to consider self-equilibrated end loads only since the nonlinearity rules out superposition and (ii) the decay rate might depend on the load level as well as on material characteristics.

The setting considered in [1] is the simplest possible within the exact theory of finite elasticity: finite anti-plane shear of an infinitely long cylinder composed of homogeneous, isotropic, incompressible material. The constitutive law is assumed to belong to the special class of such laws which permit nontrivial states of finite anti-plane shear [4]. The undeformed cross-section of the cylinder is a semi-infinite strip, the long sides of which remain traction-free while the short side carries a prescribed shear traction directed parallel to the generators. This given traction is not necessarily uniformly distributed and the associated average shear stress $\tau$ need not vanish. At infinity in the strip, the displacement is that of a simple shear with shear stress $\tau$. It is shown in [1] that, along the long sides of the strip, the difference between the nonvanishing component of shear stress and its average value $\tau$ is bounded by an exponentially decaying function of the distance from the end. A lower bound is given for the rate of decay for materials which either "harden" or "soften" in simple shear. This lower bound depends on the strip width, on the average stress $\tau$, on a measure of the departure from uniformity of the given end traction and on the strainenergy density of the material.

The foregoing results were obtained in [1] through the use of a technique based on a comparison principle for second-order quasilinear elliptic partial differential equations. The comparison function used in [1] is an eigenfunction corresponding to the linearized problem. One would expect to obtain improved estimates if an eigenfunction associated with the nonlinear problem could be utilized. We investigate this issue in the present paper.

\footnotetext{
* Received January 12, 1982. The work reported here was supported by the National Science Foundation under grants MEA-7826071 (C.O.H.) and CME8106581 (R.A.).
} 
We consider the semi-infinite strip problem described above for the special case of a self-equilibrated applied end traction $(\tau=0)$. An exponential decay inequality for the nonvanishing component of shear stress on the long sides of the strip is established. This result is qualitatively similar to that of [1] but provides a sharper estimate.

2. Finite anti-plane shear for incompressible elastic materials. Consider a body composed of an elastic material which in an undeformed state occupies an infinitely long cylinder with generators parallel to the $x_{3}$-axis. Let $\mathscr{D}$ be the (closed) cross-section of the cylinder in the plane $x_{3}=0$. An anti-plane shear of the cylinder is a deformation with a single, out-of-plane displacement component $u\left(x_{1}, x_{2}\right)$.

Suppose that the material is homogeneous, isotropic, incompressible and is characterized by an elastic potential $W$ representing the strain-energy density per unit undeformed volume. Then $W$ is a function of the first two fundamental scalar invariants $I_{1}, I_{2}$ of the Cauchy-Green deformation tensors, $W=\hat{W}\left(I_{1}, I_{2}\right)$. It is well known that not every such material can sustain a non-trivial state of anti-plane shear. Knowles [4] has determined the entire sub-class of these materials which admit such deformations and it is only this class of materials that we consider here.

It may easily be shown that in all anti-plane deformations one has $I_{1}=I_{2}(=3+$ $\left.|\nabla u|^{2}\right)$ and it is sufficient for our purposes to consider the restriction of $\hat{W}\left(I_{1}, I_{2}\right)$ to the line $I_{1}=I_{2}$. Hence we define $W(I)$ by

$$
W=W(I)=\hat{W}(I, I), \quad I \geq 3 .
$$

At infinitesimal deformations, the shear modulus is given by $\mu=2 W^{\prime}(3)>0$ where the prime denotes differentiation; $W(I)$ is assumed to be twice continuously differentiable for $I \geq 3$.

The response of this material in simple shear is described by

$$
\hat{\tau}(\gamma)=2 \gamma W^{\prime}\left(3+\gamma^{2}\right) \text { for all } \gamma,
$$

where the odd function $\hat{\tau}(\gamma)$ gives the shear stress associated with an amount of shear $\gamma$. The (secant) modulus of shear, $M$, is taken to be positive:

$$
M(\gamma) \equiv \hat{\tau}(\gamma) / \gamma=2 W^{\prime}\left(3+\gamma^{2}\right)>0 \quad \text { for all } \gamma .
$$

The components $\tau_{i j}$ of the true stress accompanying an anti-plane shear are given by (see $[4,5])$

$$
\tau_{\alpha 3}=\tau_{3 \alpha}=2 W^{\prime}(I) u_{, \alpha}, \quad \tau_{\alpha \beta}=0, \quad \tau_{33}=2 W^{\prime}(I)|\nabla u|^{2},
$$

where $I=3+|\nabla u|^{2}$. If the tractions on the lateral surface of the cylinder are independent of $x_{3}$, the field equations of finite elastostatics reduce to the following single quasilinear equation for $u$ :

$$
\left[W^{\prime}\left(3+|\nabla u|^{2}\right) u_{\alpha}\right]_{,_{\alpha}}=0 \text { on } \mathscr{D} .
$$

The traction boundary-value problem in anti-plane shear then consists of the differential equation (2.5) together with the boundary condition prescribing $\tau_{3 \alpha} n_{\alpha}=2 W^{\prime}(I) \partial u / \partial n$ on

\footnotetext{
${ }^{1}$ Greek and Latin subscripts have the respective ranges $(1,2)$ and $(1,2,3)$, repeated subscripts are summed and a subscript preceded by a comma indicates partial differentiation with respect to the corresponding $x$-coordinate.
} 
the boundary of $\mathscr{D}, \mathbf{n}$ being the unit outward normal on this boundary. At the "ends" of the cylinder $x_{3}= \pm \infty$, suitable tractions must be applied.

When (2.3) holds, the differential equation (2.5) is elliptic at a solution $u$ and at a point $\left(x_{1}, x_{2}\right)$ in $\mathscr{D}$ if and only if $\hat{\tau}^{\prime}(|\nabla u|)>0$. Thus, in particular, if the shear stress response function $\hat{\tau}(\gamma)$ is monotone strictly increasing for all $\gamma$, then (2.5) is always elliptic. Following [1], we impose a slightly stronger requirement: we assume that the shear stress response function $\hat{\tau}(\gamma)$ satisfies

$$
\gamma \hat{\tau}^{\prime}(\gamma) \geq c \hat{\tau}(\gamma) \text { for all } \gamma \geq 0,
$$

for some positive constant $c$. This, together with (2.2), (2.3), assures that (2.5) is uniformly elliptic (see [6], p. 203) and implies in particular that $\hat{\tau}^{\prime}(\gamma) \geq 0$ for all $\gamma$, as well as that $\hat{\tau}(\infty)=\infty$. Henceforth, the ellipticity constant $c$ is taken to be the largest constant for which (2.6) holds. Since (2.6) is to hold in the undeformed state $\gamma=0$, we have from (2.2), (2.3) that necessarily $c \leq 1$ and so

$$
0<c \leq 1 .
$$

It follows also that when (2.6), (2.3) hold, $\tau=\hat{\tau}(\gamma)$ can be inverted to give $\gamma$ as an odd, monotone strictly increasing function of $\tau: \gamma=\hat{\gamma}(\tau)$ with $\hat{\gamma}(\infty)=\infty$.

The main result in this paper will be given in terms of a function $\beta(s)$ related to the stress-response function $\hat{\tau}(\gamma)$ by

$$
\beta(s)=\max _{0 \leqslant \tau \leqslant s}\left(\frac{\tau}{\gamma \hat{\tau}^{\prime}(\gamma)}-1\right), \quad \gamma=\hat{\gamma}(\tau) .
$$

From (2.2) it can be shown that $\hat{\gamma}^{\prime}(\tau)=\hat{\gamma}(\tau) / \tau=1 / \mu$ as $\tau \rightarrow 0$ and so $\beta(0)=0$. Since from its definition $\beta(s)$ is non-decreasing, it follows that $\beta(s) \geq 0$. Thus in view of $(2.6)$ we conclude that

$$
0 \leq \beta(s) \leq \frac{1}{c}-1, \text { for } s \geq 0 .
$$

A particularly simple class of materials admitting a nontrivial state of anti-plane shear are those for which $W=\hat{W}\left(I_{1}\right)$. The familiar neo-Hookean strain energy density, $\hat{W}=$ $(\mu / 2)\left(I_{1}-3\right), \mu>0$, is of this form. In this case $M(\gamma)=\mu, \hat{\tau}(\gamma)=\mu \gamma$. For a neo-Hookean material, (2.5) reduces exactly to Laplace's equation, which is, of course, uniformly elliptic, and the ellipticity constant $c$ is equal to unity.

Another example in this class is furnished by the "power-law" materials introduced in [5]; here

$$
\hat{W}\left(I_{1}\right)=\frac{\mu}{2 b}\left\{\left[1+\frac{b}{n}\left(I_{1}-3\right)\right]^{n}-1\right\},
$$

where $\mu, b$ and $n$ are positive constants. The case $n=1$ corresponds to the neo-Hookean material. The associated response function in simple shear is given by

$$
\hat{\tau}(\gamma)=\mu \gamma\left(1+\frac{b}{n} \gamma^{2}\right)^{n-1} .
$$

It is easily verified that the inequality in (2.3) holds, and that (2.5) is elliptic when $n \geq \frac{1}{2}$ 
and uniformly elliptic when $n>\frac{1}{2}$. In the latter case, the ellipticity constant $c$ is given by

$$
\begin{aligned}
c & =2 n-1, & & \frac{1}{2}<n<1, \\
& =1, & & n \geq 1 .
\end{aligned}
$$

The analysis which follows applies to all materials admitting a nontrivial state of anti-plane shear which satisfy (2.3), (2.6).

3. The semi-infinite strip subject to a self-equilibrated end traction. Following [1], we now consider the case in which $\mathscr{D}$ is the semi-infinite strip $x_{1} \geq 0,0 \leq x_{2} \leq h$. The long sides are required to be free of traction, so that by (2.4)

$$
u,,_{2}\left(x_{1}, 0\right)=u,_{2}\left(x_{1}, h\right)=0, \quad x_{1} \geq 0 .
$$

The end $x_{1}=0$ is to carry a prescribed axial shear traction $\tau_{31}=f\left(x_{2}\right)$, so that, again by (2.4),

$$
2 W^{\prime}\left(3+|\nabla u|^{2}\right) u_{1}=f\left(x_{2}\right) \text { for } x_{1}=0, \quad 0 \leq x_{2} \leq h,
$$

where $f$ is a given function which is taken to be continuously differentiable for $0 \leq x_{2} \leq h$. Moreover, we restrict our attention to the case when this end traction is self-equilibrated, i.e. the resultant force (per unit axial length) on $x_{1}=0$ vanishes:

$$
\int_{0}^{h} f\left(x_{2}\right) d x_{2}=0 \text {. }
$$

At infinity it is required that the strip be stress-free, whence

$$
\begin{gathered}
u,_{1}\left(x_{1}, x_{2}\right) \rightarrow 0, \quad u,,_{2}\left(x_{1}, x_{2}\right) \rightarrow 0 \quad \text { as } \quad x_{1} \rightarrow \infty, \\
\text { uniformly in } x_{2}, \quad 0 \leq x_{2} \leq h .
\end{gathered}
$$

We assume the existence of a function $u$, continuously differentiable on $\mathscr{D}$ and twice continuously differentiable on the interior of $\mathscr{D}$, satisfying the differential equation (2.5) and the boundary conditions (3.1)-(3.4). The stress-free state, $u \equiv 0$, obeys (2.5), (3.1) and (3.4) exactly but does not satisfy (3.2) unless the given shear traction $f\left(x_{2}\right)$ is uniform (and so, by (3.3), necessarily zero). The principle of Saint-Venant type established here compares this stress-free state with that arising in the strip when the end loading is nonuniform.

In order to do this we apply, as in [1], a comparison principle for second-order quasilinear elliptic differential equations. It is convenient for this purpose to convert the foregoing problem to one of Dirichlet type. It follows from (2.4), (2.5) that there is a function $v$, with the same smoothness as $u$, which satisfies

$$
\tau_{3 \alpha}=2 W^{\prime}\left(3+|\nabla u|^{2}\right) u_{\alpha}=\varepsilon_{\alpha \beta} v_{, \beta} \text { on } \mathscr{D},
$$

where $\varepsilon_{\alpha \beta}$ is the two-dimensional alternator $\left(\varepsilon_{11}=\varepsilon_{22}=0, \varepsilon_{12}=-\varepsilon_{21}=1\right)$. The function $v$ is a stress-function for the shear stresses $\tau_{3 \alpha}$. From (3.5) and (2.2) one infers that $\hat{\tau}(|\nabla u|)$ $=|\nabla v|$, which upon inversion gives $|\nabla u|=\hat{\gamma}(|\nabla v|)$. We now define a function $V$ by

$$
1 / V\left(\tau^{2}\right)=2 W^{\prime}\left(3+\hat{\gamma}^{2}(\tau)\right)(>0) \text { for all } \tau,
$$

and observe from (2.3) that $V$ represents the shear compliance of the material. Eq. (3.5) 
may then be written as

$$
u,_{\alpha}=V\left(|\nabla v|^{2}\right) \varepsilon_{\alpha \beta} v_{,_{\beta}} .
$$

It now follows that $v$ satisfies the (elliptic) differential equation

$$
\mathscr{L} v \equiv V\left(|\nabla v|^{2}\right) v_{\alpha \alpha}+2 V^{\prime}\left(|\nabla v|^{2}\right) v,_{\alpha} v_{\beta} v_{,_{\alpha \beta}}=0 \text { on } \mathscr{D} \text {. }
$$

From (3.5), (3.1), (3.2) and (3.4) it is easy to show that the stress-function $v$ satisfies the following boundary conditions:

$$
\begin{gathered}
v\left(x_{1}, 0\right)=0, \quad v\left(x_{1}, h\right)=0, \quad x_{1} \geq 0, \\
v\left(0, x_{2}\right)=F\left(x_{2}\right) \equiv \int_{0}^{x_{2}} f(\eta) d \eta, \quad 0 \leq x_{2} \leq h, \\
v\left(x_{1}, x_{2}\right) \rightarrow 0 \text { as } x_{1} \rightarrow \infty, \quad \text { uniformly in } x_{2}, \quad 0 \leq x_{2} \leq h .
\end{gathered}
$$

Since the prescribed end traction $f$ is self-equilibrated, the function $F$ defined in (3.10) satisfies

$$
F(0)=F(h)=0 \text {. }
$$

4. Exponential decay estimates. The shear stresses are given by (3.5) as

$$
\tau_{31}=v,_{2}, \quad \tau_{32}=-v,_{1} \text { on } \mathscr{D} \text {. }
$$

The component $\tau_{32}$ vanishes on the long sides of the strip $x_{2}=0$ and $x_{2}=h$. We will show that the non-zero shear stress $\tau_{31}$ obeys an exponential decay inequality on these sides of the form

$$
\left|\tau_{31}\right| \leq q e^{-k x_{1}}, \quad x_{2}=0 \text { or } h, \quad x_{1} \geq 0 .
$$

Here the constant $q \geq 0$ is a measure of the nonuniformity of the applied traction $f\left(x_{2}\right)$, and will be described more fully subsequently. The estimated rate of decay $k$ in (4.2) (which provides a lower bound on an actual rate of decay) will be characterized in terms of $q$, the constitutive parameter $\beta$ defined in (2.8) and the strip width $h$.

The derivation of (4.2) to be presented below is based on the following comparison theorem for second-order quasilinear elliptic partial differential equations.

THEOREM. Let $v$ be a solution of the Dirichlet problem (3.8)-(3.12) on $\mathscr{D}$. Suppose that a function $\hat{v}$, with the same smoothness as $v$, exists on $\mathscr{D}$ such that

$$
\begin{gathered}
\mathscr{L} \hat{v} \leq \mathscr{L} v=0 \quad \text { on } \quad \mathscr{D}, \\
\hat{v}\left(x_{1}, 0\right)=v\left(x_{1}, 0\right)=0, \quad \hat{v}\left(x_{1}, h\right)=v\left(x_{1}, h\right)=0, \quad x_{1} \geq 0, \\
\hat{v}\left(0, x_{2}\right) \geq\left|v\left(0, x_{2}\right)\right|=\left|F\left(x_{2}\right)\right|, \quad 0 \leq x_{2} \leq h, \\
\hat{v}\left(x_{1}, x_{2}\right) \rightarrow 0 \quad \text { as } \quad x_{1} \rightarrow \infty, \quad \text { uniformly in } x_{2}, \quad 0 \leq x_{2} \leq h .
\end{gathered}
$$

2 The normalization $v(0,0)=0$ has been adopted here.

${ }^{3} F(z)$ denotes the shear force (per unit axial length) on the portion $0 \leq x_{2} \leq z$ of the end $x_{1}=0$.

${ }^{4}$ Stress estimates of the form (4.2) valid in the interior of the strip could presumably be obtained but only with considerable technical effort (cf. the remarks on p. 282 of [1]). 
Then

$$
\begin{aligned}
& \left|v\left(x_{1}, x_{2}\right)\right| \leq \hat{v}\left(x_{1}, x_{2}\right) \text { on } \mathscr{D}, \\
& \left|v,_{2}\left(x_{1}, 0\right)\right| \leq\left|\hat{v}_{2}\left(x_{1}, 0\right)\right|, \quad\left|v,_{2}\left(x_{1}, h\right)\right| \leq\left|\hat{v}_{2}\left(x_{1}, h\right)\right|, \quad x_{1} \geq 0 .
\end{aligned}
$$

Proof: This result is a special case of the theorem established on p. 284 of [1]. The proof given there makes use of a general comparison principle for second-order quasilinear elliptic equations (Theorem 9.2, p. 207 of [6]).

On recalling (4.1), we see that (4.8) provide estimates for $\tau_{31}$ on the long sides of the strip, as desired.

To facilitate the search for appropriate comparison functions $\hat{v}$ conforming to (4.3)(4.6), it proves convenient to consider a requirement on $\hat{v}$ which is different from (4.3). This essentially involves a decomposition of the differential operator $\mathscr{L}$ appearing in (4.3). By virtue of (2.2) and (3.6) it can be seen that

$$
\frac{2 V^{\prime}\left(\tau^{2}\right)}{V\left(\tau^{2}\right)}=\frac{\tau-\gamma \hat{\tau}^{\prime}(\gamma)}{\gamma \tau^{2} \hat{\tau}^{\prime}(\gamma)}, \quad \gamma=\hat{\gamma}(\tau)
$$

and so from the definition of $\beta$ in (2.8) we have

$$
\beta(s)=\max _{0 \leqslant \tau \leqslant s} \frac{2 \tau^{2} V^{\prime}\left(\tau^{2}\right)}{V\left(\tau^{2}\right)} .
$$

Consequently, it may be readily verified that, if for any positive number $s_{0}, \hat{v}$ satisfies

$$
\begin{gathered}
|\nabla \hat{v}|^{2} \hat{v}_{\alpha \alpha}+\beta\left(s_{0}\right) \hat{v}_{\alpha \alpha} \hat{v}_{\gamma} \hat{v}_{\alpha \gamma}=0 \quad \text { on } \mathscr{D}, \\
\hat{v}_{, \alpha \alpha} \leq 0, \quad 0 \leq|\nabla \hat{v}| \leq s_{0} \quad \text { on } \quad \mathscr{D},
\end{gathered}
$$

then $\mathscr{L} \hat{v} \leq 0$ on $\mathscr{D}$ provided $\beta\left(s_{0}\right)>0$. Thus we may replace (4.3) in the theorem by the simpler requirements (4.11), (4.12).

The desired result (4.2) motivates us to seek comparison functions of the form

$$
\hat{v}\left(x_{1}, x_{2}\right)=e^{-k x_{1}} G\left(x_{2}\right), \quad k>0,
$$

where $k$ and $G$ depend on the parameter $s_{0}$ in general. It now follows from the preceding discussion that if (a superposed dot denotes differentiation with respect to $x_{2}$ )

$$
\begin{gathered}
{\left[(1+\beta) \dot{G}^{2}+k^{2} G^{2}\right] \ddot{G}+k^{2}(2 \beta+1) G \dot{G}^{2}+(\beta+1) k^{4} G^{3}=0 \text { for } 0<x_{2}<h,} \\
G(0)=G(h)=0,
\end{gathered}
$$

as well as

$$
\ddot{G}+k^{2} G \leq 0, \quad \dot{G}^{2}+k^{2} G^{2} \leq s_{0}^{2}, \quad G \geq|F| \text { for } 0<x_{2}<h,
$$

then

$$
\left|\tau_{31}\left(x_{1}, 0\right)\right| \leq|\dot{G}(0)| e^{-k x_{1}}, \quad\left|\tau_{31}\left(x_{1}, h\right)\right| \leq|\dot{G}(h)| e^{-k x_{1}}, \quad x_{1} \geq 0 .
$$

Eq. (4.14) arises on substitution from (4.13) in (4.11), the first and second inequalities in (4.16) follow from (4.12), and the remaining conditions (4.15), (4.16) ${ }_{3}$ on $G\left(x_{2}\right)$ arise from (4.4), (4.5). The conclusion (4.17) follows from (4.1), (4.8) and (4.13).

It is easily shown that (4.16) ${ }_{1}$ holds automatically for all functions $G\left(x_{2}\right)$ satisfying 
(4.14), (4.16) $)_{3}$. To see this, one merely needs to observe from (4.14) that

$$
\ddot{G}+k^{2} G=-\frac{\beta k^{2}\left(\dot{G}^{2}+k^{2} G^{2}\right) G}{(\beta+1) \dot{G}^{2}+k^{2} G^{2}},
$$

and that $\beta \geq 0, G \geq 0$ according to (2.9), (4.16) ${ }_{3}$. Here and henceforth, we assume that $G$ and $\dot{G}$ do not both vanish simultaneously. Thus our task is reduced to that of determining a function $G$ satisfying (4.14), (4.15), (4.16) $2,(4.16)_{3}$.

The nonlinear eigenvalue problem (4.14), (4.15) is solved exactly in the Appendix. The smallest eigenvalue $k\left(s_{0}\right)$ is found to be

$$
k\left(s_{0}\right)=\frac{\pi}{2 h}\left(1+\frac{1}{\beta\left(s_{0}\right)+1}\right) .
$$

The corresponding eigenfunction $G$ has the form

$$
G=\frac{q}{k\left(s_{0}\right)} \hat{g}\left(x_{2}, s_{0}\right) \equiv \frac{q}{k\left(s_{0}\right)} g\left(x_{2}\right)
$$

where $q$ is an (as yet) arbitrary constant and $g\left(x_{2}\right)$ is a function whose explicit formula is given in Eq. (14) of the Appendix with $\beta=\beta\left(s_{0}\right)$. It suffices for our purposes here to note that $g\left(x_{2}\right)$ has the properties

$$
\begin{array}{cl}
g\left(x_{2}\right)>0 \quad \text { for } 0<x_{2}<h, & \dot{g}^{2}+k^{2} g^{2} \leq k^{2} \text { for } 0 \leq x_{2} \leq h, \\
g(0)=g(h)=0, & \dot{g}(0)=-\dot{g}(h)=k>0 .
\end{array}
$$

On using (4.20) and $(4.21)_{2}$ it is readily seen that $(4.16)_{2}$ holds provided

$$
q \leq s_{0} .
$$

It remains to consider the third of (4.16). By virtue of (4.20) this holds if

$$
q \geq k\left(s_{0}\right) \max _{0 \leqslant x_{2} \leqslant h} \frac{\left|F\left(x_{2}\right)\right|}{\hat{g}\left(x_{2}, s_{0}\right)} .
$$

The right-hand side of (4.24) is finite by virtue of $(4.21)_{1},(4.22)$ and the fact that $F$ is continuously differentiable and satisfies (3.12). The final estimate (4.2), with $k=k\left(s_{0}\right)$ given by (4.19), now follows (a limiting argument is required for the case $\beta\left(s_{0}\right)=0$ ) from (4.17), (4.20), (4.22) provided that the arbitrary constants $q$ and $s_{0}$ are chosen to conform to (4.23), (4.24). The selection of these constants will be discussed in the next section.

5. Results and discussion. For convenience, we rewrite here the exponential decay result established in the preceding section:

$$
\left|\tau_{31}\right| \leq q e^{-k\left(s_{0}\right) x_{1}}, \quad x_{2}=0 \quad \text { or } h, \quad x_{1} \geq 0,
$$

where

$$
\begin{gathered}
k\left(s_{0}\right)=\frac{\pi}{2 h}\left(1+\frac{1}{\beta\left(s_{0}\right)+1}\right), \\
\beta\left(s_{0}\right)=\max _{0 \leqslant \tau \leqslant s_{0}} \frac{2 \tau^{2} V^{\prime}\left(\tau^{2}\right)}{V\left(\tau^{2}\right)} .
\end{gathered}
$$


Here the constants $q$ and $s_{0}$ are to be chosen such that

$$
s_{0} \geq q \geq k\left(s_{0}\right) \max _{0 \leqslant x_{2} \leqslant h} \frac{\left|F\left(x_{2}\right)\right|}{\hat{g}\left(x_{2}, s_{0}\right)} \equiv H\left(s_{0}\right),
$$

where $\hat{g}\left(x_{2}, s_{0}\right) \equiv g\left(x_{2}\right)$ is given by Eq. (14) of the Appendix with $\beta=\beta\left(s_{0}\right)$.

A choice of $q$ and $s_{0}$ conforming to (5.4) is indeed possible, since $H\left(s_{0}\right)$ is a monotone non-increasing function of $s_{0}$, for $s_{0} \geq 0$. This follows since $\beta$ is clearly a monotone nondecreasing function of $s_{0}$ and the explicit form for $g$ and $k$ can be used to show that $H$ is monotone non-increasing with respect to $\beta$. Thus, for all sufficiently large values of $s_{0}$, there is a range of values of $q$ for which (5.4) holds.

Since $k$ is a monotone non-increasing function of $s_{0}, k\left(s_{0}\right)$ is such that $k\left(s_{0}\right) \geq k(\infty)$. From (2.9) we have $\beta(\infty)=1 / c-1$ where $c$ is the ellipticity constant and so the estimated decay rate in (5.1) has the lower bound

$$
k\left(s_{0}\right) \geq \frac{\pi}{2 h}(1+c), \quad 0<c \leq 1, \text { for } s_{0} \geq 0 .
$$

The results (5.1) $-(5.4)$ take a particularly simple form whenever $\beta \equiv 0$. This occurs, for example, when $V^{\prime}\left(\tau^{2}\right) \leq 0$, which corresponds to materials which do not soften in simple shear, i.e. the modulus of shear is such that $M^{\prime}(\gamma) \geq 0$. A second example is a material for which the ellipticity constant $c=1$ (see 2.9). When $\beta \equiv 0,(5.2)$ yields

$$
k=\pi / h \text {. }
$$

Moreover, from Eqs. (11) and (14) of the Appendix with $\beta=0$, it follows that $g\left(x_{2}\right)=\sin$ $\left(\pi x_{2} / h\right)$ and thus $H\left(s_{0}\right)$ in (5.4) is independent of $s_{0}$. The smallest value of $q$ consistent with (5.4) is then $q=Q$, where

$$
Q=\frac{\pi}{h} \max _{0 \leqslant x_{2} \leqslant h} \frac{\left|F\left(x_{2}\right)\right|}{\sin \left(\pi x_{2} / h\right)} .
$$

If the anti-plane shear is infinitesimal $(|\nabla u| \ll 1)$, the differential equation $(2.5)$ reduces to Laplace's equation upon linearization about the undeformed state. For the resulting linearized boundary value problem, it can be shown (see [1], pp. 282-283) that the estimate (5.1) holds, with $k, q=Q$ given by (5.6), (5.7) respectively. The estimated decay rate (5.6) is the best possible one for the linearized problem. ${ }^{5}$

In what follows we make two particular choices of the pair $\left(s_{0}, q\right)$ satisfying (5.4). In the first of these, we obtain the smallest possible value of the multiplicative factor $q$ in the estimate (5.1). For this purpose we take $s_{0}=\infty$ and $q=H(\infty)$. Thus

$$
k=k(\infty)=\frac{\pi}{2 h}(1+c), \quad 0<c \leq 1 .
$$

The corresponding value of $q$ is

$$
q=H(\infty)=\frac{\pi}{2 h}(1+c) \max _{0 \leqslant x_{2} \leqslant h} \frac{F\left(x_{2}\right)}{\hat{g}\left(x_{2}, \infty\right)},
$$

\footnotetext{
$s$ These observations remain true for a neo-Hookean material, without any linearization.
} 
where

$$
\begin{gathered}
\hat{g}\left(x_{2}, \infty\right)=\cos \psi\left(x_{2}\right) \exp \left\{\frac{(c-1)}{2} \cos ^{2} \psi\left(x_{2}\right)\right\}, \\
x_{2}=\frac{h}{2}+\frac{h}{\pi}\left\{\left(\frac{1-c}{1+c}\right) \frac{\sin 2 \psi\left(x_{2}\right)}{2}-\psi\left(x_{2}\right)\right\}, \quad-\frac{\pi}{2} \leq \psi \leq \frac{\pi}{2}, \quad 0<c \leq 1 .
\end{gathered}
$$

The second choice of the pair $\left(s_{0}, q\right)$ that we consider is such that the estimated decay rate $k$ in (5.1) is as large as possible. For this we take the unique choice given by $s_{0}=$ $H\left(s_{0}\right), q=s_{0}>0$. Thus the result in this case can be written as

$$
\begin{gathered}
\left|\tau_{31}\right| \leq q e^{-k(q) x_{1}}, \quad x_{2}=0 \quad \text { or } \quad h, \quad x_{1} \geq 0, \\
k(q)=\frac{\pi}{2 h}\left(1+\frac{1}{\beta(q)+1}\right), \quad q=H(q),
\end{gathered}
$$

where $\beta(q)$ is given by (5.3). The characterization of the estimated decay rate in (5.13), though furnishing a superior bound, is clearly more complicated than that of (5.8).

An exponential decay result of the form (5.1) has also been established in [1] under more restrictive constitutive assumptions than those of the present paper. In addition to (2.3), (2.6) the materials considered there are assumed to either soften or harden in shear, i.e. either $M^{\prime}(\gamma)<0$ or $M^{\prime}(\gamma)>0$ for all $\gamma$. For hardening materials, the results of [1] coincide with those of the present paper (see (5.1), (5.6), (5.7)). For softening materials the estimate given in [1] reads

$$
\begin{gathered}
\left|\tau_{31}\right| \leq Q e^{-K(Q) x_{1}}, \quad x_{2}=0 \text { or } h, \quad x_{1} \geq 0, \\
K(Q)=\frac{\pi}{h}\left(\frac{1}{1+Q^{2} \phi(Q)}\right)^{1 / 2}, \\
\phi(Q)=\max _{0 \leqslant \tau \leqslant Q} \frac{2 V^{\prime}\left(\tau^{2}\right)}{V\left(\tau^{2}\right)}>0,
\end{gathered}
$$

with $Q$ given by (5.7).

We now show that for softening materials

$$
q \leq Q, \quad k(q)>K(Q),
$$

so that the estimate (5.12), (5.13) obtained in the present paper is sharper than the result in [1]. The first of (5.17) may be verified from $(5.13)_{2}$ on using the monotonicity of $H$ and observing that $H(0)=Q$. To see that the second of $(5.17)$ holds, we first recall that $\beta$ is monotone non-decreasing and so $k(q) \geq k(Q)$. Next, from the definitions of $\beta$ in (5.3) and of $\phi$ in (5.16) we have that $\beta(Q) \leq Q^{2} \phi(Q)$ and so (5.13) and $_{1}$ (5.15) yield ${ }^{7} k(Q)>K(Q)$. This completes the verification of $(5.17)_{2}$.

Thus the estimated decay rates obtained here and in [1] have been shown to depend in general on a nonuniformity parameter $q$ as well as on constitutive parameters and on the strip width. As we have seen in the present paper, less sharp estimates on the decay rate, which are independent of $q$, can also be obtained (see Eq. (5.5)).

${ }^{6}$ See Eqs. (6.2), (6.3), (7.4), (7.6) and (7.20) of [1] with some minor notation changes made here.

${ }^{7}$ This makes use of the fact that, for softening materials, $\beta\left(s_{0}\right)>0$ (see Eq. (5.3) where $V^{\prime}>0$ ). 
Finally, it should be emphasized that the results obtained here pertain to the case of a semi-infinite strip subjected to a self-equilibrated applied end traction. Whether the analysis given here can be modified to account for non-self-equilibrated loads remains an open question.

Appendix: Solution of the nonlinear eigenvalue problem (4.14), (4.15). Our objective here is the solution of the eigenvalue problem

$$
\begin{gathered}
{\left[(1+\beta) \dot{G}^{2}+k^{2} G^{2}\right] \ddot{G}+k^{2}(2 \beta+1) G \dot{G}^{2}+(\beta+1) k^{4} G^{3}=0,0<x_{2}<h,} \\
G(0)=G(h)=0,
\end{gathered}
$$

for the smallest eigenvalue $k>0$ and the corresponding eigenfunction $G\left(x_{2}\right)$, the constant $\beta$ being positive. With this end in mind we introduce polar coordinates $(\rho, \psi)$ in the phase-plane of $G$ by setting

$$
k G\left(x_{2}\right)=\rho\left(x_{2}\right) \cos \psi\left(x_{2}\right), \quad \dot{G}\left(x_{2}\right)=\rho\left(x_{2}\right) \sin \psi\left(x_{2}\right), \quad 0 \leq x_{2} \leq h .
$$

The compatibility of (3) requires that

$$
\dot{\rho} \cos \psi=(k+\psi) \rho \sin \psi,
$$

whereas (1) and (3) lead to

$$
(\beta+1) \dot{\rho} \sin \psi=-k(\beta+1) \rho \cos \psi-\psi \rho \cos \psi .
$$

Elimination of $\dot{\rho}$ between Eqs. (4) and (5) yields

$$
\psi=-\frac{k(\beta+1)}{\beta \sin ^{2} \psi+1} \quad(<0) .
$$

The boundary conditions (2) and the first of (3) require that $\cos \psi(0)=\cos \psi(h)=0$. Since, according to (6), $\psi\left(x_{2}\right)$ is monotone strictly decreasing for $0 \leq x_{2} \leq h$, we now choose

$$
\psi(h)=-\frac{\pi}{2}, \quad \psi(0)=(2 N+1) \frac{\pi}{2} \quad(N=0,1, \ldots) .
$$

Integration of (6) together with the first of (7) leads to

$$
x_{2}=h-\frac{\pi}{4 k} \frac{\beta+2}{\beta+1}+\frac{\beta+2}{2 k(\beta+1)}\left[\frac{\beta}{2(\beta+2)} \sin 2 \psi-\psi\right] .
$$

It is easily seen that (8) is consistent with the second of the boundary conditions (7) if and only if

$$
k=\frac{\pi}{h} \frac{\beta+2}{2(\beta+1)}(N+1) .
$$

The smallest eigenvalue $k$ is thus obtained from (9) with $N=0$ and is

$$
k=\frac{\pi}{h} \frac{\beta+2}{2(\beta+1)} \text {. }
$$

${ }^{8}$ The introduction of phase-plane coordinates is suggested by the fact that (1) is autonomous. Knowles and Sternberg [7] have previously used phase-plane representations to study similar nonlinear eigenvalue problems arising in crack problems in finite elastostatics. 
We are now left with determining the corresponding eigenfunction $G$. We note first that (8), (10) imply

$$
x_{2}=\frac{h}{2}+\frac{h}{\pi}\left\{\frac{\beta}{2(\beta+2)} \sin 2 \psi-\psi\right\}, \quad-\frac{\pi}{2} \leq \psi \leq \frac{\pi}{2} .
$$

It is readily shown that (11) is uniquely invertible, leading to a function $\psi\left(x_{2}\right)$ defined for $0 \leq x_{2} \leq h$. Next, we have from (3) that $\dot{G}=k \tan \psi G$, which on using (6) gives

$$
\frac{d G}{d \psi}=-\left\{\beta \sin ^{2} \psi \tan \psi+\tan \psi\right\} \frac{G}{(\beta+1)} .
$$

Integration of (12) gives the eigenfunction $G\left(x_{2}\right)$ as

$$
\begin{gathered}
G\left(x_{2}\right)=\frac{q}{k} g\left(x_{2}\right), \\
g\left(x_{2}\right)=\cos \psi \cdot \exp \left\{\frac{-\beta}{2(\beta+1)} \cos ^{2} \psi\right\},
\end{gathered}
$$

with $\psi=\psi\left(x_{2}\right)$ given by (11). Alternatively, one may view (11) and (14) as being a parametric definition of the function $g\left(x_{2}\right)$. Here $q$ is an arbitrary constant.

The following properties of the function $g\left(x_{2}\right)$ readily follow from (11) and (14):

$$
\begin{gathered}
g\left(x_{2}\right)>0 \text { for } 0<x_{2}<h, \\
\dot{g}\left(x_{2}\right)>0 \text { for } 0 \leq x_{2}<\frac{h}{2}, \quad \dot{g}\left(x_{2}\right)<0 \text { for } \frac{h}{2}<x_{2} \leq h, \\
\dot{g}(0)=k, \quad \dot{g}(h)=-k .
\end{gathered}
$$

Furthermore, it can be shown on using (3), (4), (6) and (15) that

$$
\dot{g}^{2}+k^{2} g^{2} \leq k^{2} \text { for } 0 \leq x_{2} \leq h .
$$

\section{REFERENCES}

[1] C. O. Horgan and J. K. Knowles, The effect of nonlinearity on a principle of Saint-Venant type, J. Elasticity 11, 271-291 (1981)

[2] C. O. Horgan and J. K. Knowles, Recent developments concerning Saint-Venant's principle, in Advances in Applied Mechanics 23 (J. W. Hutchinson, ed.), Academic Press, New York (in press)

[3] M. E. Gurtin, The linear theory of elasticity, in Handbuch der Physik, (S. Flügge, ed.), Vol VI a/2, Springer, Berlin, 1972, pp. 1-295

[4] J. K. Knowles, On finite anti-plane shear for incompressible elastic materials, J. Austral. Math. Soc. (series B) 19, 400-415 (1976)

[5] J. K. Knowles, The finite anti-plane shear field near the tip of a crack for a class of incompressible elastic solids, Int. J. Fracture 13, 611-639 (1977)

[6] D. Gilbarg and N. S. Trudinger, Elliptic partial differential equations of second order, Springer-Verlag, Berlin, 1977

[7] J. K. Knowles and E. Sternberg, An asymptotic finite-deformation analysis of the elastostatic field near the tip of a crack, J. Elasticity 3, 67-107 (1973) 